\title{
Multicell Coordination via Joint Scheduling, Beamforming and Power Spectrum Adaptation
}

\author{
Wei Yu \\ Dept. of Electrical and Computer Eng. \\ University of Toronto \\ Toronto, Ontario, Canada M5S 3G4 \\ Email: weiyu@comm.utoronto.ca
}

\author{
Taesoo Kwon and Changyong Shin \\ New Radio Access Group, Communication Lab \\ Samsung Electronics \\ Gyeonggi-Do, South Korea, 446-712 \\ Emails: \{taesoo.kwon, c.y.shin\}@samsung.com
}

\begin{abstract}
The mitigation of intercell interference is a central issue for future generation wireless cellular networks where frequencies are reused aggressively and where hierarchical cellular structures may heavily overlap. The paper examines the benefit of coordinating transmission strategies and resource allocation schemes across multiple cells for interference mitigation. For a multicell network serving multiple users per cell sectors and where both the base-stations and the remote users are equipped with multiple antennas, this paper proposes a joint proportionally fair scheduling, spatial multiplexing, and power spectrum adaptation method that coordinates multiple basestations with an objective of optimizing the overall network utility. The proposed scheme optimizes the user schedule, transmit and receive beamforming vectors, and transmit power spectra jointly, while taking into consideration both the intercell and intracell interference and the fairness among the users. The proposed system is shown to significantly improve the overall network throughput while maintaining fairness as compared to a conventional network with per-cell zero-forcing beamforming and with fixed transmit power spectrum. The proposed system goes toward the vision of a fully coordinated multicell network, whereby transmission strategies and resource allocation schemes (rather than transmit signals) are coordinated across the basestations as a first step.
\end{abstract}

\section{OVERVIEW}

\section{A. Introduction}

Intercell interference is a fundamental limiting factor in wireless cellular networks. A promising idea for the mitigation of intercell interference in future cellular networks is the coordination of multiple base-stations. In a fully coordinated multicell system, multiple antennas across multiple basestations can be thought of as forming a large antenna array, where intercell interference can be actively exploited. The realization of such a fully coordinated system, however, also requires high-capacity backhaul communication. As antennas from across multiple base-stations need to jointly transmit and receive signals for multiple mobile users, data streams of multiple users must be shared among multiple base-stations.

This paper explores a different type of coordination where user transmission strategies and resource allocation schemes, rather than data signals, are coordinated across the basestations. The coordination of transmission strategies clearly requires much less backhaul communication, and is much easier to implement in a practical deployment. The goal of this paper is to show that by jointly setting the scheduling, power allocation, and beamforming strategies of multiple basestations and multiple mobile users within each cell, intercell interference can already be alleviated, and the overall performance of the network can already be improved significantly as compared to the current generation of wireless networks where cells operate independently.

Resource management has been the focus of extensive studies for cellular networks in the past, but traditional studies typically focus on per-cell strategies. This is in part due to the fact that coordination across the multiple cells presents a significant challenge not only from an implementation point of view, but also in optimization, as the presence of intercell interference leads to inherent nonconvexity in the problem structure. This paper adopts a network utility maximization framework and makes progress on these accounts. We show that network-wide optimization can be performed on each of the scheduling, beamforming, and power allocation modules separately and iteratively, and that distributed implementation is possible with reasonable amount of intercell messaging. We utilize ideas such as interference pricing for multicell power spectrum adaptation and uplink-downlink duality for coordinated beamforming to devise an efficient and distributed optimization algorithm that is capable of reaching a networkwide (albeit local) optimum. A main contribution of this paper is a multiuser scheduling algorithm that naturally takes channel gain, orthogonality, intercell interference, and user traffic demand into account. In addition, we provide realistic simulations results to quantify the benefit of multicell resource management. While previous works in this area typically focus on the performance evaluation of specific optimization components (e.g. power control, scheduling, or beamforming) individually, this paper takes a system-wide approach and analyzes the interaction among them. We show that under realistic cellular deployment scenarios, the coordination of transmission and resource allocation strategies across multiple cells brings significant throughput benefit to users at the cell edge, and an overall utility improvement to the entire network.

\section{B. System Model}

This paper considers a wireless multiple-input multipleoutput (MIMO) multicell network such as the one shown in 


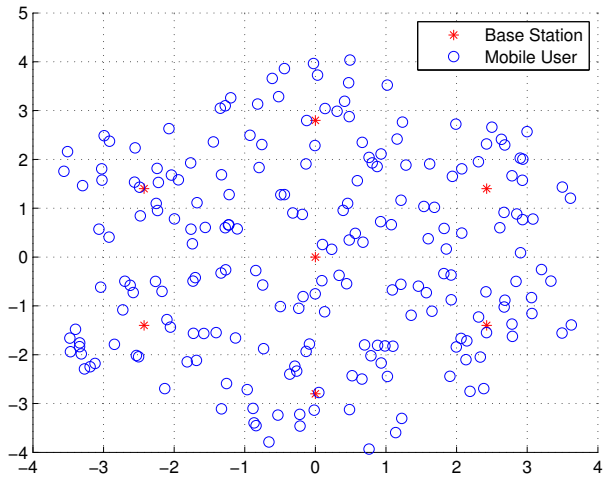

Fig. 1. A Cellular topology with 7 cells, 3 sectors per cell, and 10 users per sector placed near the cell edge.

Fig. 1, where both the base-stations and the mobile users are equipped with multiple antennas and where multiple users within each cell are separated either in frequency via orthogonal frequency-division multiple-access (OFDMA), or in timeslots via scheduling, or via spatial multiplexing via beamforming. We assume that the network employs an initial channel estimation and synchronization phase, in which the MIMO multipath fading channels between every pair of transmitter and receiver are estimated across the frequency tones. This includes both uplink and downlink direct channels within each cell as well as the interfering channels between any pair of transmitter and receiver (either base-station or remote terminal) in neighboring cells.

This paper aims to tackle the following network-wide resource allocation question. Given the total amount of time, frequency and spatial resources in each cell, how should they be distributed across the users to maximize the total network utility? This question is important in future wireless systems, as modern networks are increasingly deployed with maximal frequency reuse and with hierarchical structure where cells can heavily overlap. Consequently, intercell (and intersector) interference often becomes the dominant limiting factor. In addition, because of the use of spatial multiplexing where multiple users are served in the same time/frequency slot simultaneously, mobile users can also experience intracell interference. Thus, the above resource allocation problem is coupled both across the users within each cell and across the cells. The goal of this paper is to devise efficient optimization techniques that strike a balance between maximizing each user's own data rate and minimizing the effect of its interference on its neighbors-an task that needs to be facilitated by multicell coordination.

\section{Problem Statement}

Consider a wireless cellular MIMO-OFDMA network with spatial multiplexing within each cell, where multiple basestations coordinate in their resource allocation strategies, but otherwise transmit and receive data streams independently, the a joint scheduling, spatial multiplexing, and power spectrum adaptation problem can be stated as follows:
1) Beamforming: What are the appropriate transmit and receive beamforming vectors at the base-stations and at the mobile users?

2) Scheduling: Which user should be served in each frequency and time slots for each beam?

3) Power spectrum allocation: What is the appropriate power spectrum for each beam?

In general, these three questions must be answered jointly. Further, the optimization must be performed repeatedly over time as channels vary, and a separate optimization procedure must be performed for each of the uplink and the downlink.

This papers adopts a proportional fairness objective across the users, i.e. the optimization procedure aims to maximize

$$
\max \sum_{l s k} \log \left(\bar{R}_{l s k}\right)
$$

where $\bar{R}_{l s k}$ is the long term average rate of the $k$ th user in the $l$ th cell and the sth sector. The transmit signals are subject to transmit power spectral density (PSD) constraints across the antennas at either the base-stations for the downlink, or the mobile terminals for the uplink.

\section{Proposed Approach}

This paper adopts an iterative optimization approach to solve the above problem. Our key observation is that the three questions above can be decoupled and solved in an iterative fashion. Specifically, the proposed solution involves the following steps:

- Fixing the assignment of users and power for each transmit beam, the update of the beamforming vectors can be done in a coordinated fashion across the cells via a concept known as uplink-downlink duality;

- Fixing the beamforming vectors and power allocation, the assignment of users to each beam can be done in a greedy fashion via proportionally fair scheduling;

- Fixing the beamformers and user assignment, the power updates can be coordinated across the cells via interference pricing.

The above three steps can be iterated to reach a (local) optimal solution of the joint optimization problem. Such a solution allows multiple cells to coordinate in alleviating intercell interference, thereby improving the overall network utility.

\section{E. Related Work}

Scheduling, beamforming and power allocation methods have been the subject of extensive studies in the singlecell multiuser MIMO environment. For example, joint power allocation and scheduling is considered in [1], [2], [3] and the use of joint zero-forcing beamforming and user scheduling is considered in [4], [5], [6], [7]. In addition to zero-forcing beamforming, eigen-mode beamforming [8], gradient-based beamforming [9], and other heuristic interference-aware beamforming designs [10], [11] have also been considered in the literature. From a more rigorous perspective, a concept known as uplink-downlink duality has emerged as a key solution to the problem of finding the optimal beamforming vectors to 
minimize transmit power subject to signal-to-interference-andnoise-ratio (SINR) constraints [12], [13], [14], [15], [16], [17], [18], [19], [20]. The use of this duality-based beamforming, together with power control and branch-and-bound heuristic scheduling has been considered in [21]. The joint scheduling, power allocation and beamforming problem also appears in the multi-hop literature [22], [23], and for ad-hoc networks [24].

In the multicell context, scheduling, beamforming and power allocation have mostly been studied separately in the past. For example, the joint design of beamforming across multiple cells has been considered in [25], [26], [27], [28], [29], [30], [31], [32], but these studies typically focus on the minimization of transmit power for a fixed set of selected users, instead of the optimization of network utility. Likewise, intercell scheduling has been considered in [33], [34], [35], [36], [37], [38], and intercell power control has been considered in [39], [40], but these studies do not include beamforming.

The proposed approach in this study is novel in that it offers an optimization framework for the overall multicell multiuser MIMO system, in contrast to previous studies which only focus on a subset of the design variables. For example, joint scheduling and zero-forcing beamforming is studied in [41], and joint scheduling and opportunistic beamforming is studied [42], but both without power optimization, while [43], [44] consider beamforming with power allocation, but without scheduling. Finally, [45], [46], [47], [48], [49] consider joint scheduling and power control, but without beamforming,

The joint optimization of scheduling, beamforming and power allocation is a challenging problem mathematically. For example, despite the many beamforming techniques that are available, the problem of selecting the best set of active users within a sector is combinatorial in nature, and is thought to be difficult to solve. In addition, the optimization of power and beamformers (with fixed user selection) is another well-known and difficult nonconvex problem. In this paper, we propose a way to incrementally update user selection for each fixed beamforming and power allocation. This gives a graceful way of dealing with the combinatorial nature of the scheduling problem, while allowing practical and locally optimal methods to be used for power control, thereby providing a reasonably good solution for the overall system.

The proposed system treats the beamforming problem using the uplink-downlink duality technique, and treats the scheduling problem using a proportionally fair scheduler [50]. In addition, power spectrum adaptation is performed using a concept called interference pricing [51], [52], [53], [54], [55], which allows the effect of interference among multiple transmitter-receiver pairs to be quantified. One of the novelties of this paper is the use of the Newton's method for fast convergence in power adaptation.

It is worth emphasizing that the proposed system allows multiple cells to coordinate in their signaling strategy (e.g. power, beamforming and scheduling), but not in the actual data streams. We show that transmission strategy and resource allocation coordination already brings significant improvement to existing cellular system [56]. This is a first step toward the vision of a network MIMO system with user signal-level coordination, which would approach the ultimate capacity limit of cellular networks [57], [58], [59], [60], [61], [62].

\section{Algorithm}

\section{A. Mathematical Formulation}

Consider an interference-limited multicell environment with $L$ cells, $S$ sectors per cell, $K$ users per sector, and an OFDMA multiplexing scheme with $N$ tones over a fixed bandwidth. The base-station is equipped with $P$ antennas, and the remote users are equipped with $Q$ antennas each. Let $H_{l s, m t k}^{n}$ denote the $P \times Q$ matrix channel between the $l$ th base-station, the $s$ th sector, and the $k$ th remote user in the $m$ th cell, the $t$ th sector for both uplink and downlink in tone $n$. The system is assumed to operate in a time-division duplex (TDD) mode.

The proposed system uses a spatial multiplexing scheme so that each base-station may serve up to $P$ users simultaneously, but each user is assigned at most one data stream in each given tone. While in a single-user MIMO channel, having multiple data streams for a user is advantageous from a capacity point of view, one data stream per user is sensible in a multiuser environment where multiuser diversity ensures that such a restriction is near optimal. Further, we assume that the basestation does not employ nonlinear interference presubtraction (i.e. dirty-paper coding). In this case, the $P$ users in the downlink are separated by linear transmit beamforming vectors $v_{D, l s b}^{n}$, which denotes the $b$ th downlink transmit beamformer in the $l$ th cell and $s$ th sector, and linear receive beamforming vectors $u_{D, l s k}^{n}$, which denotes the downlink receive beamformer applied at the $k$ th mobile user in the $l$ th cell, the $s$ th sector. The beamforming vectors have unit norm. The notation for the uplink is similar.

A key issue in the OFDMA system is user scheduling. We use an assignment function $f_{D}(l, s, b, n)$ to assign a user $k$ to the $b$ th beamformer in the $l$ th cell, the $s$ th sector, the $n$th tone in the downlink, and likewise $f_{U}(l, s, b, n)$ for the uplink.

Let $P_{U, l s b}^{n}$ and $P_{D, l s b}^{n}$ be the uplink and downlink transmit PSDs in the $l$ th cell, the $s$ th sector, the $b$ th beamformer, the $n$th tone, at the assigned remote user for uplink and at the base-station for downlink, respectively. The downlink proportionally fair joint scheduling, beamforming, and transmit power spectrum adaptation problem is that of choosing the user scheduling function $k=f_{D}(l, s, b, n)$, the beamforming vectors $v_{D, l s b}^{n}$ and $u_{D, l s k}^{n}$, and the downlink transmit power $P_{D, l s b}^{n}$ to maximize (1), i.e.

$$
\begin{array}{ll}
\max & \sum_{l, s, k} \log \left(\bar{R}_{D, l s k}\right) \\
\text { s.t. } & R_{D, l s k}=\sum_{\substack{\left\{(b, n): k=f_{D}(l, s, b, n)\right\}\\
}} \log \left(1+\mathrm{SINR}_{D, l s b k}^{n}\right) \\
& 0 \leq P_{D, l s b}^{n} \leq S_{D}^{\max } \quad \forall l, s, b, n
\end{array}
$$


where

$$
\begin{aligned}
& \operatorname{SINR}_{D, l s b k}^{n}= \\
& \frac{P_{D, l s b}^{n}\left|\left(u_{D, l s k}^{n}\right)^{T} H_{l s, l s k}^{n} v_{D, l s b}^{n}\right|^{2}}{\Gamma\left(\sigma^{2}+\sum_{(j, t, c) \neq(l, s, b)} P_{D, j t c}^{n}\left|\left(u_{D, l s k}^{n}\right)^{T} H_{j t, l s k}^{n} v_{D, j t c}^{n}\right|^{2}\right)}
\end{aligned}
$$

Here, $\bar{R}_{D, l s k}$ is the time averaged rate and $R_{D, l s k}$ is the instantaneous downlink rate for the $k$ th user in the $l$ th cell and the $s$ th sector. The uplink problem formulation is similar. Note that the SINR expression includes the intracell interference due to the power leakage from other transmit beams within each sector as well as intercell interference coming from neighboring cells or neighboring sectors. Finally, $\Gamma$ is the SNR gap accounting for the realistic choices of modulation and coding schemes; $\sigma^{2}$ is the background noise.

The above formulation contains a simplifying modeling assumption that a peak power constraint $S_{D}^{\max }$ is imposed on each beamforming vector separately. A more realistic constraint would be a constraint on the transmit power of each antenna element. The simplified per-beam power constraint used here leads to considerable optimization simplicity, while retaining the essential feature of the optimal solution.

The optimization problems (2) is a mixed discrete (user scheduling) and continuous (beamforming and power allocation) optimization problem. This paper proposes an approach based on iteratively solving the scheduling, beamforming, and power allocation subproblems.

\section{B. Proportionally Fair Scheduling with Spatial Multiplexing}

A key question in the design of spatial multiplexing systems is that of selecting the set of active users in each cell/sector and for each frequency tone. Clearly, it is desirable to schedule users whose channels are nearly orthogonal. In addition, the scheduler also needs to balance the user traffic demand and the individual user channel gains. Solving this problem optimally would requires a combinatorial search, which is clearly not feasible in practice. This paper proposes a novel approach of gracefully switching users in and out of the active set using proportionally fair scheduling. The idea is that instead of selecting the best set of users then designing beamforming vectors for them, we iteratively fix the beamformers and select the users according to the proportionally fairness criterion, then update the beamformers assuming a fixed user selection.

The proposed user selection strategy relies on the following key observation. In the downlink, the interference produced by each beamformer to users both within its own sector and in neighboring sectors is a function of the beamformer and its associated transmit power only, and is independent of the user assignment at this beam. Thus, at the $l$ th cell, the $s$ th sector, and the $b$ th beam, if the beamforming vector $v_{D, l s b}^{n}$ and the power allocation $P_{D, l s b}^{n}$ are fixed, user scheduling can be done independently in each cell on a per-beam basis without affecting the interference level elsewhere in the network. This enables a simple search algorithm for finding the user that maximizes the proportional fairness objective: for each beamforming vector, the algorithm finds the user who benefits the most from being scheduled in that beamformer:

$$
f_{D}(l, s, b, n)=\operatorname{argmax}_{k} \frac{r_{D, l s k}^{n}}{\bar{R}_{D, l s k}}
$$

where $\bar{R}_{k}$ is the long-term average rate updated exponentially with some $0<\alpha<1$ as follows

$$
\bar{R}_{D, l s k}=\alpha \bar{R}_{D, l s k}+(1-\alpha) R_{D, l s k}
$$

where $r_{D, l s k}^{n}$ is the instantaneous rate in frequency tone $n$ computed from the fixed power spectrum allocation as in (2). The above scheduling policy maximizes log utility as the derivative of the $\log$ utility is $1 / \bar{R}_{D, l s k}$. The scheduling policy (4) is essentially the solution to a weighted rate sum maximization problem with weights chosen as $1 / \bar{R}_{D, l s k}$.

The proposed algorithm can be thought of as a MIMO extension of the joint scheduling and power control algorithm proposed in [48], [49], [45] for the OFDMA network, where the scheduling step is done in each frequency tone.

A key novelty of the proposed policy is that scheduling is done on a per-beam basis, so it naturally takes the intercell interference and the channel orthogonality of the spatial multiplex system into account through the computation of SINR for each beam. The proposed scheduling policy also naturally accounts for the temporally varying channels and user traffic demands. As the channels and consequently the associated achievable rate region vary over time, different users are scheduled to account for the different user priorities, fairness, channel gains and orthogonality, and intercell interference.

The proposed scheduling policy depends critically on the fact that the user assignment at each beam does not affect the interference elsewhere in the network. But, this is true only for the downlink, and not for the uplink. However, in this paper, we propose to use the same scheduling policy for both uplink and downlink in a TDD system as in [45]. This can be justified by uplink-downlink duality, i.e. under the same sum power constraint, the uplink and downlink capacity regions are the same. Although practical networks are not sum-power constrained, system-level simulation nevertheless shows that this approach gives reasonable performance.

Finally, we remark that the proposed scheduling policy (4) can be implemented in each cell/sector in a distributed fashion as intercell interference can be easily measured locally.

\section{Beamforming}

It remains to find the optimal beamforming vector and the optimal power allocation for the fixed active user set. The proportional fairness objective gives rise to the following downlink weighted rate sum maximization problem

$$
\max \sum_{l s k} w_{D, l s k} R_{D, l s k}, \quad \text { where } \quad w_{D, l s k}=\frac{1}{\bar{R}_{D, l s k}}
$$

over the power and beamforming vectors. This problem is known to be difficult to solve because of its underlying nonconvex problem structure. Here, we again propose a separated approach, i.e. iteratively finding a set of good beamforming 
vectors for fixed power allocation, then finding a set of good power allocations for fixed beamformers. This section deals with the beamforming design first.

One sensible approach for beamforming design is to set the beamforming vectors so that the interference within each sector is completely nulled out. This is known as zero-forcing (ZF) beamforming. When each mobile user is equipped with a single antenna, downlink ZF beamforming is equivalent to channel inversion. In a MIMO setting where mobile users have multiple antennas, it is possible to iterate between setting the minimum mean squared error (MMSE) receive beamformer at the mobiles and ZF transmit beamformer at the base-station to reach a simultaneous ZF and MMSE solution.

However, ZF is a per-cell (or per-sector) strategy, which does not take into account intercell interference. Our goal here is to develop coordinated beamforming strategies across the base-stations so that intercell interference may be mitigated.

The proposed strategy is based on a fact known as "uplinkdownlink duality". For a multicell multiuser system where base-stations are equipped with multiple antennas but mobile users are equipped with a single antenna each, under a fixed set of SINR constraints, the power-minimizing downlink transmit beamformers at the base-station are exactly the MMSE receive beamformers of a dual uplink sum-power minimizing network. This duality relationship holds not only for single-cell systems [16], [17], [18], [14], [15] but also for multicell systems as shown in [12], [28], [26].

Previous uses of this duality relationship have been restricted to the minimization of transmit sum power across the network. The main novelty here is the integration of this power minimization step in an overall framework for utility maximization. The proposed coordinated beamforming (CBF) strategy for a multicell multiuser MIMO downlink system is as follows. Assuming a fixed downlink power allocation and user selection, for every tone $n$ :

1) Initialize a set of downlink transmit beamformers $v_{D, l s b}^{n}$;

2) Find and fix the optimal MMSE downlink receive beamformers $u_{D, l s k}^{n}$, so the mobile users can now effectively be regarded as single-antenna users;

3) Compute the current set of SINR's for every user;

4) Form the virtual dual uplink channel by taking the conjugate transpose of all the channel matrices, and iterate between the following two steps:

a) Find the appropriate power in the virtual dual uplink channel to satisfy the current set of SINR's. This can be done via a matrix inversion, or using an iterative power update (see e.g. [28]).

b) Find the MMSE receive beamformers in the virtual dual uplink for the given virtual uplink power.

5) Set the downlink transmit beamformers $v_{D, l s b}^{n}$ to be the virtual dual uplink receive beamformer;

6) Find the downlink power to satisfy the current set of SINR's;

7) Update the downlink receive beamformers $u_{D, l s k}^{n}$ as the optimal MMSE receive beamformers;
8) Go to Step (4). Iterate until convergence.

An identical algorithm can be implemented in the uplink to find the optimal uplink transmit and receive beamformers.

The above algorithm finds the appropriate beamforming vectors that minimize the total transmit power in the entire multicell network, subject to the SINR constraints at each user. Essentially, this part of the algorithm finds the optimal beamformers for the fixed SINRs, while relying on the additional user scheduling and power allocation steps to find the optimal set of SINRs for utility maximization. Note that the algorithm iteratively updates the transmit and receive beamformers. Each of these iterative steps reduces the total transmit power, so the iteration is guaranteed to converge.

It is possible to implement this beamforming step in a distributed fashion [28]. This is because by the reciprocity property of wireless electromagnetic propagation, the uplink and downlink wireless channel matrices are in fact conjugate transposes of each other. Note that the implementation of uplink-downlink duality in real channels requires the implementation of virtual dual uplink (and downlink) powers. Therefore, as long as there is a beamforming update phase where all the users temporarily implement the virtual powers for beamforming updates, distributed implementation of the proposed $\mathrm{CBF}$ algorithm is possible.

\section{Dynamic Power Spectrum Adaptation}

The third component of the overall algorithm is a power spectrum adaptation step, assuming a fixed user schedule and a fixed set of beamformers. The objective is again the weighted rate sum maximization problem (6). The optimization variables are the per-beam transmit PSDs. As the beamforming vectors are fixed, the optimization is essentially on a set of point-to-point interfering links. As mentioned earlier, a perbeam (i.e. per link) power constraint is imposed for simplicity.

The optimization of transmit power for weighted rate-sum maximization is a difficult problem with no known convex formulation. Existing approaches typically rely on convex approximation (e.g. [63], [64]), but global optimality is difficult to establish. Nevertheless, this paper makes an observation that local optimality often already brings in significant improvement to existing systems. Further, there are efficient and distributed methods for reaching these local optimal points.

This paper advocates an ascent approach based on Newton's method. The implementation of the algorithm relies on the passing of messages among the multiple base-station. The idea is to coordinate the PSDs of multiple beams in multiple cells via messages which are functions of the proportional fairness variables in each cell and the transmit PSDs, signal-to-noise ratios, direct and interfering channel gains for each beam. The messages summarize the effect of interference each beam causes to its neighbors.

The use of interference pricing has appeared in previous works, but mostly for single-antenna systems [51], [55], [52], [49], [45]. The present work applies the idea to multicell multiantenna beamforming systems. In addition, this paper uses a Newton direction for faster convergence as proposed in [45]. 
Consider first the downlink. The optimization problem can be decomposed into $N$ independent problems, one per each tone $n=1, \cdots, N$ :

$$
\begin{array}{ll}
\max & \sum_{l, s, b} w_{D, l s k} r_{D, l s k}^{n} \\
\text { s.t. } & 0 \leq P_{D, l s b}^{n} \leq S_{D}^{\max }
\end{array}
$$

where

$$
r_{D, l s k}^{n}=\log \left(1+\frac{P_{D, l s b}^{n}\left|h_{l s b, l s k}^{n}\right|^{2}}{\Gamma\left(\sigma^{2}+\sum_{(j t c) \neq(l s b)} P_{D, j t c}^{n}\left|h_{j t c, l s k}^{n}\right|^{2}\right)}\right)
$$

with $k=f_{D}(l, s, b, n)$ and $\left|h_{j t c, l s k}^{n}\right|^{2}=\left|\left(u_{l s k}^{n}\right)^{T} H_{j t, l s k}^{n} v_{j t c}^{n}\right|^{2}$. The idea is to define an interference price for each beam at each base-station and each tone as follows:

$$
\begin{aligned}
t_{D, j t c, l s b}^{n} & =w_{D, j t k^{\prime}} \frac{\partial r_{D, j t k^{\prime}}^{n}}{\partial P_{D, l s b}^{n}} \\
& =w_{D, j t k^{\prime}} \frac{\Gamma\left|h_{l s b, j t k^{\prime}}^{n}\right|^{2}}{P_{D, j t c}^{n}\left|h_{j t c, j t k^{\prime}}^{n}\right|^{2}} \frac{\left(\operatorname{SINR}_{D, j t c}^{n}\right)^{2}}{1+\operatorname{SINR}_{D, j t c}^{n}}(9)
\end{aligned}
$$

where

$$
\operatorname{SINR}_{D, j t c}^{n}=\frac{P_{D, j t c}^{n}\left|h_{j t c, j t k^{\prime}}^{n}\right|^{2}}{\Gamma\left(\sigma^{2}+\sum_{(l s b) \neq(j t c)} P_{D, l s b}^{n}\left|h_{l s b, j t k^{\prime}}^{n}\right|^{2}\right)},
$$

and $k^{\prime}=f_{D}(j, t, c, n)$. The interference price $t_{D, j t c, l s b}$ quantifies the effect of power allocation at the $l$ th base-station, the $s$ th sector and the $b$ th beam to the data rate of the user served by the $j$ th base-station, the $t$ th section, and the $c$ th beam.

We can now use the gradient and an approximate Hessian of the objective function to form a Newton's direction for power update. To simplify the computation, only the diagonal elements of the Hessian matrix are used. The power update direction is

$$
\Delta P_{D, l s b}^{n}=\frac{\frac{w_{D, l s k}}{P_{D, l s b}^{n}}\left(1+\frac{1}{\operatorname{SINR}_{D, l s b}^{n}}\right)^{-1}-\sum_{(j t c) \neq(l s b)} t_{D, j t c, l s b}^{n}}{\frac{w_{D, l s k}}{\left(P_{D, l s b}^{n}\right)^{2}}\left(1+\frac{1}{\operatorname{SINR}_{D, l s b}^{n}}\right)^{-2}}
$$

where $k=f_{D}(l, s, b, n)$. The numerator in (11) is the gradient of the objective function with respect to $P_{D, l s b}^{n}$. The denominator is the corresponding diagonal term of the Hessian. The interference pricing terms enter the gradient computation.

In summary, each beam in each cell and each sector iteratively updates its power allocation according to

$$
P_{D, l s b}^{n}(\kappa+1)=\left[P_{D, l s b}^{n}(\kappa)+\mu \Delta P_{D, l s b}^{n}\right]_{0}^{S_{D}^{\max }} .
$$

As the Newton's direction is an ascent direction, the convergence can be easily ensured with an appropriate step size $\mu$. An identical algorithm can be implemented for the uplink. This power allocation step can be implemented in a distributed fashion with the exchange of interference pricing variables $t_{D, j t c, l s b}^{n}$ between the base-stations.

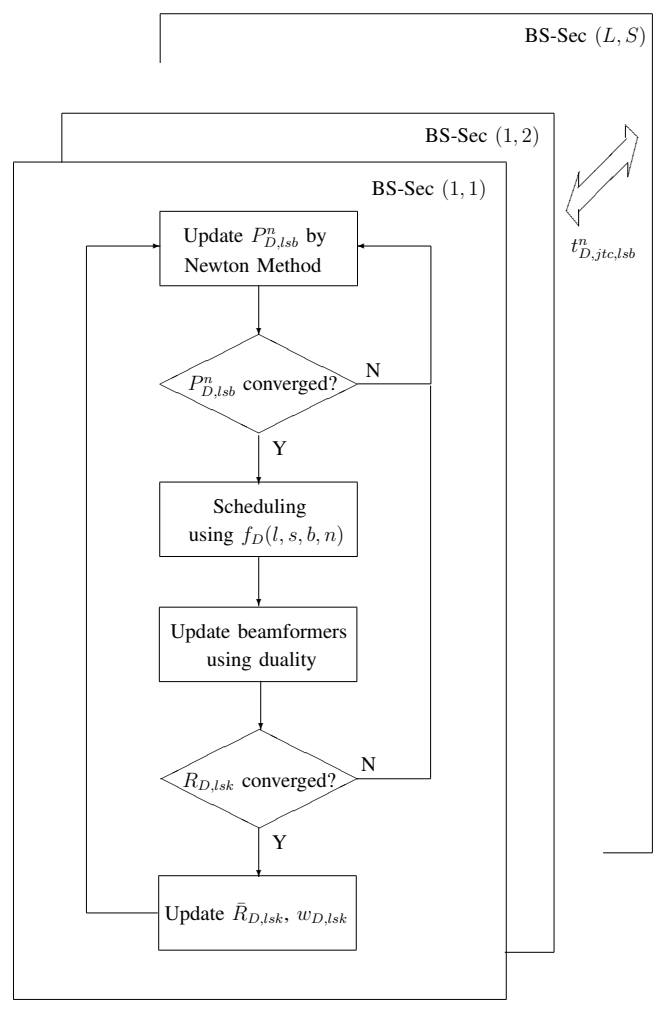

Fig. 2. The joint proportionally fair scheduling, adaptive beamforming, and power spectrum adaptation algorithm for downlink. The uplink algorithm is similar.

\section{E. Summary of the Algorithm}

The user scheduling, beamforming, and power spectrum adaptation steps are iterated until convergence. Each step is a nondecreasing step in network utility. Thus, the iterations converge to at least a local optimum solution. The entire algorithm is depicted in Fig. 2.

\section{Performance Projection}

The performance of the proposed algorithm is evaluated on a wireless cellular network with 7 cells, 3 sectors per cell, and 10 users per sector, with maximal frequency reuse, and where cells are wrapped around so that each cell has six neighboring cells. The base-station is equipped with 4 antennas, allowing 4 users to be served simultaneously in each frequency tone. The remote users are equipped with 2 antennas. System parameters are outlined in Table I. The users are distributed randomly in each cell as shown in Fig. 1. The base-station-to-base-station distance is $2.8 \mathrm{~km}$ corresponding to a typical WiMax or LTE deployment. Frequency selective channels with a Rayleigh fading component are simulated. Perfect channel estimation is assumed. For evaluation purposes, the channels are assumed fixed throughout. Both uplink and downlink scenarios are simulated.

The algorithm is initialized with uniform power allocation at maximum PSD level of $-27 \mathrm{dBm} / \mathrm{Hz}$ per beamforming vector for both uplink and downlink, so that over a $10 \mathrm{MHz}$ bandwidth 


\begin{tabular}{|c|c|}
\hline Cellular Layout & $\begin{array}{c}\text { Hexagonal, 7 cells } \\
3 \text { sectors/cell }\end{array}$ \\
\hline BS-to-BS Distance & $2.8 \mathrm{~km}$ \\
\hline Frequency Reuse & 1 \\
\hline Number of users per sector & 10 \\
\hline Duplex & TDD \\
\hline Channel Bandwidth & $10 \mathrm{MHz}$ \\
\hline BS Max Tx Power & $49 \mathrm{dBm}$ \\
\hline BS Max Per-Beam PSD & $-27 \mathrm{dBm} / \mathrm{Hz}$ \\
\hline MS Max Per-Beam PSD & $-27 \mathrm{dBm} / \mathrm{Hz}$ \\
\hline Antenna Gain & $15 \mathrm{dBi}$ \\
\hline SNR Gap (with coding) & $6 \mathrm{~dB}$ \\
\hline Background Noise & $-169 \mathrm{dBm} / \mathrm{Hz}$ \\
\hline Noise Figure & $7 \mathrm{~dB}$ \\
\hline BS Tx Antenna No. & 4 \\
\hline MS Rx Antenna No. & 2 \\
\hline Number of beamformers at BS & 4 \\
\hline Multipath Time Delay Profile & ITU-R M.1225 PedA \\
\hline Distance-dependent path loss & $128.1+37.6 \log _{10}(d)$ \\
\hline FFT Size & 64 \\
\hline
\end{tabular}

TABLE I

WIRELESS CELLULAR MODEL PARAMETERS the total transmit power at the base-station is at $49 \mathrm{dBm}$. The initial user assignment and beamformers are set randomly.

Table II shows the achieved sum rates, Fig. 3 shows the achieved $\log$ utility, and Fig. 4 shows the cumulative distribution function of user rates for a simulation of the 7 cells with either ZF or coordinated beamforming and with and without the dynamic power spectrum adaptation (DP). Without dynamic power spectrum adaptation, both uplink and downlink transmitters simply transmit at the maximum constant PSD level. The results show that

- Dynamic power spectrum adaptation always outperforms constant power allocation in terms of both log utility and the average sum rate.

- Coordinated beamforming always outperforms zeroforcing both in log utility and the average sum rate.

- Dynamic power adaptation alone achieves a higher utility than coordinated beamforming alone in the uplink.

- Combined dynamic power spectrum adaptation and coordinated beamforming produces $10 \%-30 \%$ sum rate increase for the entire network.

- Combined dynamic power spectrum adaptation and coordinated beamforming produces the most significant rate improvement for cell edge users as seen in Fig. 4. In the downlink, it produces $100 \%$ rate improvement for the 25 th percentile users, and $50 \%$ rate improvement for the 40th percentile users. In the uplink, it produces $100 \%$ rate improvement for the 40th percentile users.

Fig. 5 illustrates the convergence of the per-sector sum rates with the joint scheduling, beamforming and power allocation algorithm. The convergence speed is reasonably fast.

\begin{tabular}{|l|c|c|}
\hline Sum Rate over 7 Cells & DL & UL \\
\hline \hline Constant PSD, Zero Forcing & 932 Mbps & $1092 \mathrm{Mbps}$ \\
\hline Constant PSD, Coord. BF & $1140 \mathrm{Mbps}$ & $1223 \mathrm{Mbps}$ \\
\hline Dynamic PSD, Zero Forcing & $1025 \mathrm{Mbps}$ & $1129 \mathrm{Mbps}$ \\
\hline Dynamic PSD, Coord. BF & $1194 \mathrm{Mbps}$ & $1230 \mathrm{Mbps}$ \\
\hline \hline Improvement & $28 \%$ & $13 \%$ \\
\hline
\end{tabular}

TABLE II

IMPROVEMENT IN SUM RATE OVER 7 CELLS, 3 SECTORS PER CELL, 10 USERS PER SECTOR. CELL DIAMETER IS $2.8 \mathrm{KM}$.

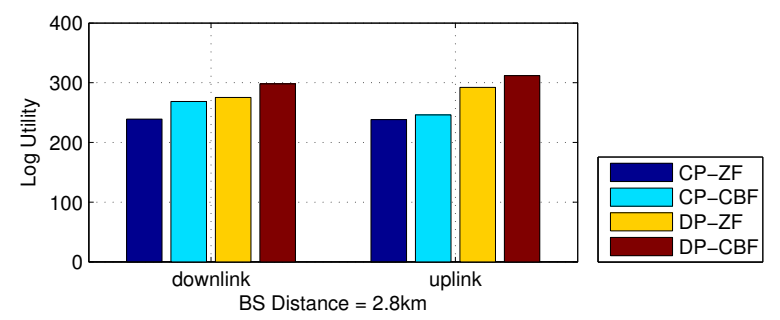

Fig. 3. Log utility gain due to dynamic power adaptation (DP) vs constant power spectrum (CP), and zero-forcing (ZF) vs. coordinated beamforming (CBF). The sum log utility is taken over 7 cells, 3 sectors per cell, and 10 users per sector. The rates are in Mbps.

\section{CONCLusions}

This paper proposes a coordinated scheduling, beamforming, and power allocation scheme across the multiple basestations in a wireless cellular network. The novelty of the proposed optimization approach lies in the decoupling of the scheduling, beamforming, and power allocation steps, and in novel application of ideas such as uplink-downlink duality and interference pricing based power control. The proposed system achieves a significant throughput and network utility improvement with coordination only at the resource allocation level and not in data signals, which is attractive especially for future deployments with heavily overlapped cellular structures.

\section{REFERENCES}

[1] X. Liu, E. K. P. Chong, and N. B. Shroff, "Joint scheduling and powerallocation for interference management in wireless networks," in IEEE Veh. Tech. Conf. (VTC-Fall), 2002, vol. 3, pp. 1892-1896.

[2] F. She, W. Chen, H. Luo, T. Huang, and X. Wang, "Joint power allocation and scheduling of multi-antenna OFDM system in broadcast channel," in IEEE Int. Conf. Commun. (ICC), 14-18 2009, pp. 1-5.

[3] T. H. Kim and S. Choi, "Interference mitigation via scheduling for the MIMO broadcast channel with limited feedback," in IEEE Int. Symp. Personal, Indoor and Mobile Radio Communications (PIMRC), 13-16 2009, pp. 2035-2039.

[4] J. Wang, D. J. Love, and M. D. Zoltowski, "User selection with zeroforcing beamforming achieves the asymptotically optimal sum rate," IEEE Trans. Signal Processing, vol. 56, no. 8, pp. 3713-3726, Aug. 2008.

[5] V. K. N. Lau and Y.-K. Kwok, "Performance analysis of SIMO space-time scheduling with convex utility function: Zero-forcing linear processing," IEEE Trans. Veh. Technol., vol. 53, no. 2, pp. 339-350, Mar. 2004.

[6] Y. Hara, L. Brunel, and K. Oshima, "Spatial scheduling with interference cancellation in multiuser MIMO systems," IEEE Trans. Veh. Technol., vol. 57, no. 2, pp. 893-905, Mar. 2008. 

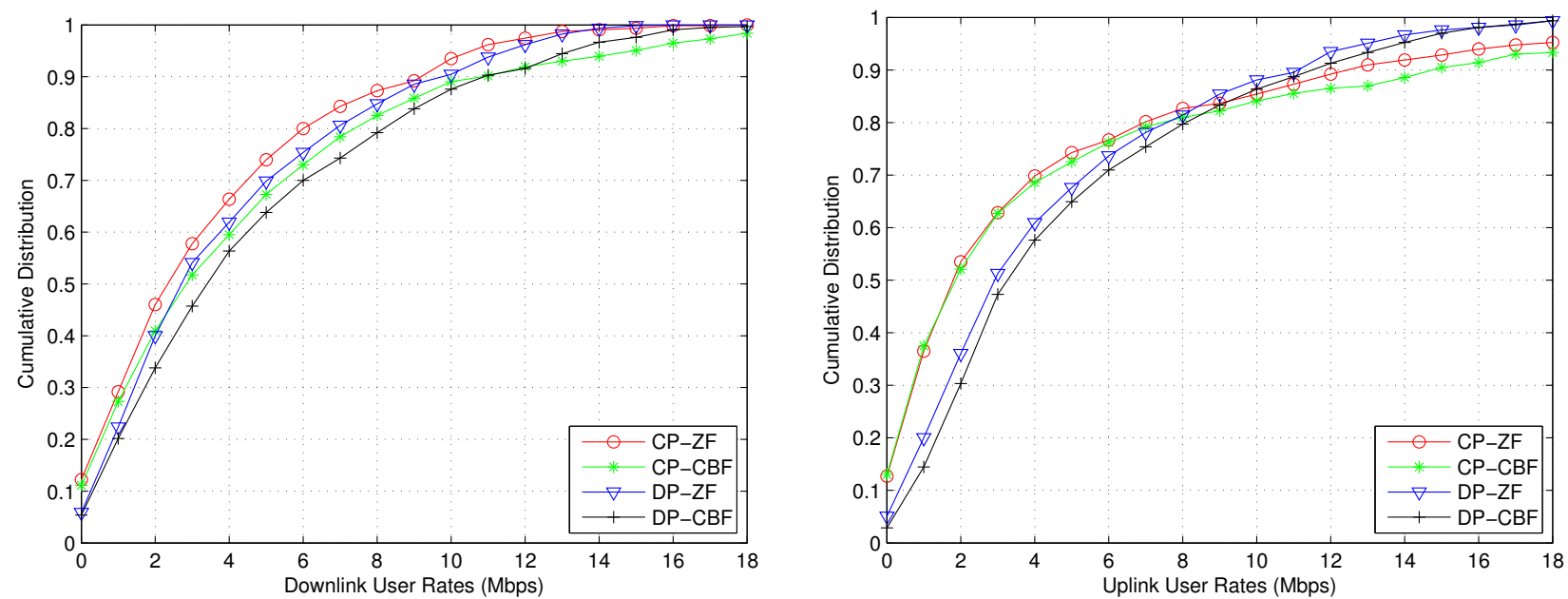

Fig. 4. Cumulative distribution function of user rates with dynamic power spectrum adaptation (DP) vs constant power (CP), and coordinated beamforming (CBF) vs zero-forcing (ZF). Cell diameter is $2.8 \mathrm{~km}$
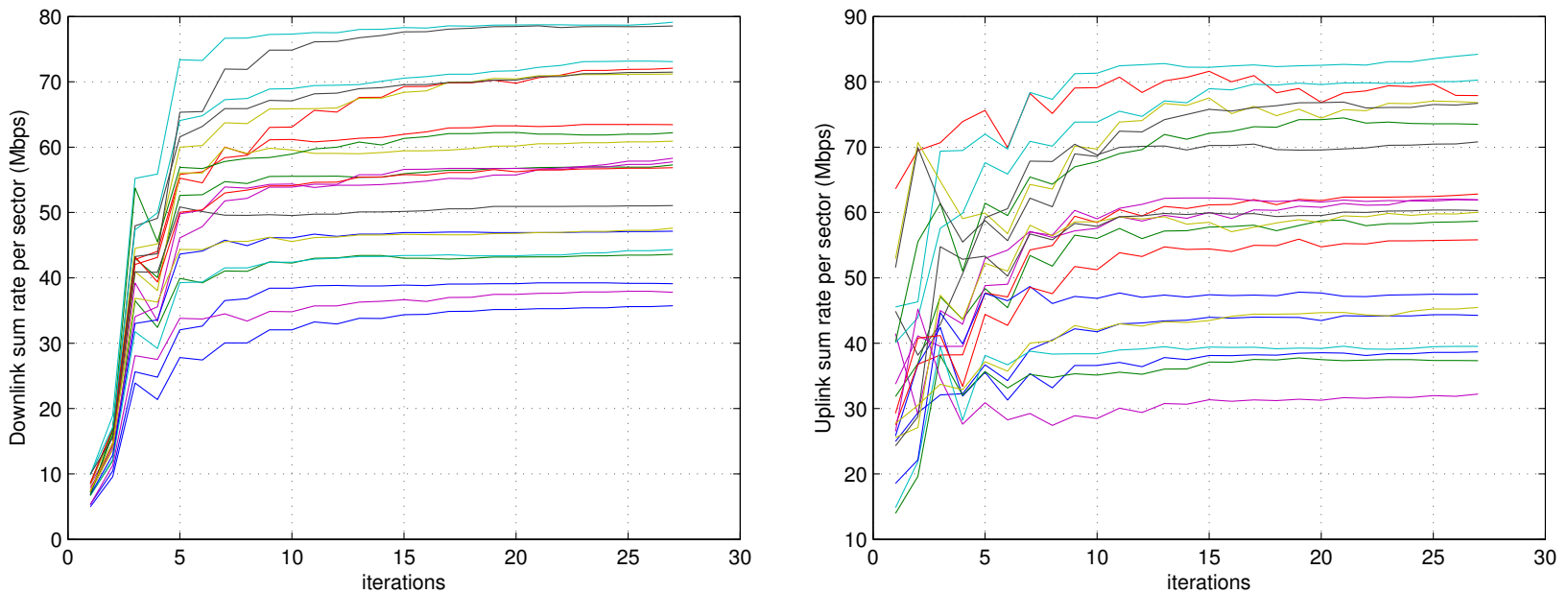

Fig. 5. Convergence of downlink (left) and uplink (right) per-sector sum rates in each of the 21 sectors with dynamic spectrum allocation and coordinated beamforming. Each iteration is either a joint user scheduling and beamforming step, or a power allocation step. Each beamforming step consists of fixed 3 inner downlink transmit beamformer and power iterations and 5 outer downlink receive beamformer updates (for a total of 15 iterations.) Each power allocation step involves up to 10 iterations. The proportional fairness weights are also updated at the same time.

[7] J. Mundarath, P. Ramanathan, and B. Van Veen, "A distributed downlink scheduling method for multi-user communication with zero-forcing beamforming," IEEE Trans. Wireless Commun., vol. 7, no. 11, pp. 45084521, Nov. 2008.

[8] M. Kountouris, R. de Francisco, D. Gesbert, D. T. M. Slock, and T. Salzer, "Low complexity scheduling and beamforming for multiuser MIMO systems," in IEEE Workshop on Signal Processing Advances in Wireless Communications (SPAWC), 2-5 2006, pp. 1-5.

[9] M. Kobayashi and G. Caire, "Joint beamforming and scheduling for a multi-antenna downlink with imperfect transmitter channel knowledge," IEEE J. Select. Areas Commun., vol. 25, no. 7, pp. 1468-1477, Sept. 2007.

[10] J. He and M. Salehi, "Low-complexity coordinated interference-aware beamforming for MIMO broadcast channels," in IEEE Veh. Tech. Conf. (VTC-Fall), sept. 2007, pp. 685-689.

[11] E. Conte, S. Tomasin, and N. Benvenuto, "A simplified greedy algorithm for joint scheduling and beamforming in multiuser MIMO OFDM," IEEE Commun. Lett., vol. 14, no. 5, pp. 381-383, May 2010.

[12] B. Song, R. Cruz, and B. Rao, "Network duality for multiuser MIMO beamforming networks and applications," IEEE Trans. Commun., vol. 55, no. 3, pp. 618-630, Mar. 2007
[13] M. Codreanu, A. Tolli, M. Juntti, and M. Latva-aho, "Joint design of tx-rx beamformers in MIMO downlink channel," IEEE Trans. Signal Processing, vol. 55, no. 9, pp. 4639-4655, sept. 2007.

[14] M. Schubert and H. Boche, "Solution of the multiuser downlink beamforming problem with individual SINR constraints," IEEE Trans. Veh. Technol., vol. 53, pp. 18-28, Jan. 2004.

[15] M. Schubert and H. Boche, "Iterative multiuser uplink and downlink beamforming under SINR contraints," IEEE Trans. Signal Processing, vol. 53, pp. 2324-2334, July 2005.

[16] F. Rashid-Farrokhi, K. J. R. Liu, and L. Tassiulas, "Transmit beamforming and power control for cellular wireless systems," IEEE J. Select. Areas Commun., vol. 16, no. 8, pp. 1437-1450, Oct. 1998.

[17] F. Rashid-Farrokhi, L. Tassiulas, and K. J. R. Liu, "Joint optimal power control and beamforming in wireless networks using antenna arrays," IEEE Trans. Commun., vol. 46, no. 10, pp. 1313-1324, Oct. 1998.

[18] E. Visotsky and U. Madhow, "Optimal beamforming using transmit antenna arrays," in Proc. IEEE Veh. Technol. Conf., July 1999, vol. 1, pp. 851-856.

[19] A. Wiesel, Y. C. Eldar, and S. Shamai, "Linear precoding via conic optimization for fixed MIMO receivers," IEEE Trans. Signal Processing, vol. 54, no. 1, pp. 161-176, Jan. 2006 
[20] W. Yu and T. Lan, "Transmitter optimization for the multi-antenna downlink with per-antenna power constraints," IEEE Trans. Signal Processing, vol. 55, no. 6, pp. 2646-2660, June 2007.

[21] B. Song, Y.-H. Lin, and R. L. Cruz, "Weighted max-min fair beamforming, power control, and scheduling for a MISO downlink," IEEE Trans. Wireless Commun., vol. 7, no. 2, pp. 464-469, Feb. 2008.

[22] J. Kim, X. Lin, and N. B. Shroff, "Locally optimized scheduling and power control algorithms for multi-hop wireless networks under SINR interference models," in Int. Symp. Modeling and Optimization in Mobile, Ad Hoc and Wireless Networks and Workshops (WiOpt), 16-20 2007, pp. 1-10.

[23] B. Song and R. L. Cruz, "Joint beamforming, power control and scheduling for multihop wireless networks," in IEEE Military Communications Conference (MILCOM), 13-16 2003, vol. 2, pp. 1005-1010.

[24] Y. Rong and Y. Hua, "Space-time power scheduling of MIMO links fairness and qos considerations," IEEE J. Select. Areas Commun., vol. 2, no. 2, pp. 171-180, Apr. 2008.

[25] R. Stridh, M. Bengtsson, and B. Ottersten, "System evaluation of optimal downlink beamforming with congestion control in wireless communication," IEEE Trans. Wireless Commun., vol. 5, pp. 743-751, Apr. 2006

[26] J. Yang and D. K. Kim, "Multi-cell uplink-downlink beamforming throughput duality based on Lagrangian duality with per-base station power constraints," IEEE Commun. Lett., vol. 12, no. 4, pp. 277-279, Apr. 2008

[27] M. Ku and D. Kim, "Tx-rx beamforming with multiuser MIMO channels in multiple-cell systems," in Proc. IEEE Inter. Conf. Advanced Commun. Tech. (ICACT), Feb. 2008, vol. 3, pp. 1767-1771.

[28] H. Dahrouj and W. Yu, "Coordinated beamforming for the multi-cell multi-antenna wireless systems," IEEE Trans. Wireless Commun., vol. 9, no. 5, pp. 1748-1759, May 2010.

[29] R. Zakhour, Z. K. M. Ho, and D. Gesbert, "Distributed beamforming coordination in multicell MIMO channels," in Proc. IEEE Veh. Tech. Conf., Barcelona, Spain, Apr. 2009.

[30] E. Larsson and E. Jorswieck, "Competition versus cooperation on the MISO interference channel," IEEE J. Select. Areas Commun., vol. 26, no. 9, pp. 1059-1069, Sept. 2008.

[31] B. O. Lee, H. W. Je, I. Sohn, O.-S. Shin, and K. B. Lee, "Interferenceaware decentralized precoding for multicell MIMO TDD systems," in IEEE Global Telecommun. Conf. (GLOBECOM), Nov. 2008, pp. 1-5.

[32] C. Botella, G. Pinero, A. Gonzalez, and M. de Diego, "Coordination in a multi-cell multi-antenna multi-user w-cdma system: A beamforming approach," IEEE Trans. Wireless Commun., vol. 7, pp. 4479-4485, Nov. 2008.

[33] W. Choi and J. G. Andrews, "The capacity gain from intercell scheduling in multi-antenna systems," IEEE Trans. Wireless Commun., vol. 7, no. 2, pp. $714-725$, Feb. 2008

[34] C. F. Binder, R. Veronesi, and V. Tralli, "Downlink scheduling with adaptive antennas in multicell SDMA packet access networks," in IEEE Veh. Tech. Conf. (VTC-Spring), 30 2005, vol. 3, pp. 1908-1912.

[35] S. G. Kiani and D. Gesbert, "Optimal and distributed scheduling for multicell capacity maximization," IEEE Trans. Wireless Comm., vol. 7 , no. 1, pp. 288-297, Jan. 2008.

[36] R. Bendlin, Y.-F. Huang, M. T. Ivrlac, and J. A. Nossek, "Fast distributed multi-cell scheduling with delayed limited-capacity backhaul links," in IEEE Int. Conf. Commun. (ICC), 14-18 2009, pp. 1-5.

[37] P. Hosein, "Cooperative scheduling of downlink beam transmissions in a cellular network," in IEEE Global Telecommun. Conf. (GLOBECOM), Nov. 2008, pp. 1-5.

[38] T. Ren and R. J. La, "Downlink beamforming algorithms with inter-cell interference in cellular networks," IEEE Trans. Wireless Commun., vol. 5, pp. 2814-2823, Oct. 2006

[39] D. Gesbert, S. G. Kiani, A. Gjendemsj, and G. E. Ien, "Adaptation, coordination, and distributed resource allocation in interference-limited wireless networks," Proc. of the IEEE, vol. 95, no. 5, pp. 2393-2409, Dec. 2007.

[40] A. Gjendemsjo, G.E. Oien, and D. Gesbert, "Binary power control for multi-cell capacity maximization," in IEEE Workshop on Signal Processing Advances in Wireless Communications (SPAWC), 17-20 2007, pp. $1-5$.

[41] H.J. Bang, "Multicell zero-forcing and user scheduling on the downlink of a linear cell-array," in IEEE Workshop on Signal Processing Advances in Wireless Communications (SPAWC), 21-24 2009, pp. 156-160.
[42] M. Vemula, D. Avidor, J. Ling, and C. Papadias, "Inter-cell coordination, opportunistic beamforming and scheduling," in IEEE Int. Conf. Commun. (ICC), June 2006, vol. 12, pp. 5319-5324.

[43] R. Veronesi, J. Zander, V. Tralli, M. Zorzi, and F. Berggren, "Distributed dynamic resource allocation for multicell SDMA packet access networks," in IEEE Int. Conf. Commun. (ICC), June 2004, vol. 1, pp. 202-207.

[44] L. Venturino, N. Prasad, and X. Wang, "Coordinated linear beamforming in downlink multi-cell wireless networks," IEEE Trans. Wireless Commun., vol. 9, no. 4, pp. 1451 -1461, Apr. 2010.

[45] W. Yu, T. Kwon, and C. Shin, "Joint scheduling and dynamic power spectrum optimization for wireless multicell networks," in Conference on Information Science and Systems (CISS), Princeton, NJ, Mar. 2010.

[46] J. Huang, V. G. Subramanian, R. Agrawal, and R. Berry, "Downlink scheduling and resource allocation for OFDM systems," in Conference Info. Science Sys. (CISS), Mar. 2006, pp. 1271-1279.

[47] J. Huang, V. G. Subramanian, R. Agrawal, and R. Berry, "Joint scheduling and resource allocation in uplink OFDM systems for broadband wireless access networks," IEEE J. Sel. Top. Signal Processing, vol. 27, no. 2, pp. 226-234, Feb. 2009.

[48] L. Venturino, N. Prasad, and X. Wang, "Coordinated scheduling and power allocation in downlink multicell OFDMA networks," IEEE Trans. Veh. Technol., vol. 58, no. 6, pp. 2835-2848, July 2009.

[49] A. L. Stolyar and H. Viswanathan, "Self-organizing dynamic fractional frequency reuse for best-effort traffic through distributed inter-cell coordination," in INFOCOM, Apr. 2009.

[50] E. F. Chaponniere, P. J. Black, J. M. Holtzman, and D. N. C. Tse, "Transmitter directed, multiple receiver system using path diversity to equitably maximize throughput," U.S. Patent 6,449,490, filed July 1999.

[51] J. Huang, R. A. Berry, and M. L. Honig, "Distributed interference compensation for wireless networks," IEEE J. Select. Areas Commun., vol. 24, no. 5, May 2006.

[52] J. Yuan and W. Yu, "Distributed cross-layer optimization of wireless sensor networks: A game theoretic approach," in Global Telecommunications Conf. (GLOBECOM), San Francisco, U.S.A., 2006.

[53] C. Shi, R. A. Berry, and M. L. Honig, "Distributed interference pricing for OFDM wireless networks with non-separable utilities," in Conference Info. Science Sys. (CISS), Mar. 2008, pp. 755-760.

[54] F. Wang, M. Krunz, and S. Cui, "Price-based spectrum management in cognitive radio networks," IEEE J. Sel. Top. Signal Processing, vol. 1, no. 2, pp. 74-87, Feb. 2008.

[55] W. Yu, "Multiuser water-filling in the presence of crosstalk," in Inform. Theory and Appl. Workshop (ITA), San Diego, U.S.A., Jan. 2007.

[56] W. Choi and J. Andrews, "Downlink performance and capacity of distributed antenna systems in a multicell environment," IEEE Trans. Wireless Commun., vol. 6, no. 1, pp. 69-73, Jan. 2007.

[57] M. Karakayali, G. Foschini, and R. Valenzuela, "Network coordination for spectrally efficient communications in cellular systems," IEEE Wireless Commun., vol. 13, no. 4, pp. 56-61, Aug. 2006.

[58] S. Shamai and B. Zaidel, "Enhancing the cellular downlink capacity via co-processing at the transmitting end," in Proc. IEEE Veh. Technol. Conf., 2001, vol. 3, pp. 1745-1749.

[59] G. Foschini, M. Karakayali, and R. Valenzuela, "Coordinating multiple antenna cellular networks to achieve enormous spectral efficiency," IEE Proc. Cummun., vol. 153, no. 4, pp. 548-555, Aug. 2006.

[60] O. Simeone, O. Somekh, G. Kramer, S. Shamai, and H. V. Poor, "Throughput of cellular systems with conferencing mobiles and cooperative base stations," EURASIP Journal on Wireless Commun. and Networking, vol. 2008, Article ID 652325, 14 pages, 2008. doi: $10.1155 / 2008 / 652325$

[61] H. Zhang and H. Dai, "Cochannel interference mitigation and cooperative processing in downlink multicell multiuser MIMO networks," EURASIP Journal on Wireless Commun. and Networking, vol. 2004, no. 2, pp. 222-235, Dec. 2004, Article ID 202654.

[62] S. Jing, D. N. C. Tse, J. B. Soriaga, J. Hou, J. Smee, and R. Padovani, "Multicell downlink capacity with coordinated processing," EURASIP Journal on Wireless Commun. and Networking, vol. 2008, no. 5, Jan. 2008, Article ID 586878.

[63] M. Chiang, C. W. Tan, D. P. Palomar, D. O'Neill, and D. Julian, "Power control by geometric programming," IEEE Trans. Wireless Commun., vol. 6, no. 7, pp. $2640-2651$, July 2007.

[64] J. Papandriopoulos and J.S. Evans, "Scale: A low-complexity distributed protocol for spectrum balancing in multiuser dsl networks," IEEE Trans. Inform. Theory, vol. 55, no. 8, pp. 3711-3724, Aug. 2009. 